University of Warwick institutional repository: http://go.warwick.ac.uk/wrap This paper is made available online in accordance with publisher policies. Please scroll down to view the document itself. Please refer to the repository record for this item and our policy information available from the repository home page for further information.

To see the final version of this paper please visit the publisher's website. Access to the published version may require a subscription.

Author(s): XAVIER BUFF and ADAM L. EPSTEIN

Article Title: From local to global analytic conjugacies

Year of publication: 2009

Link to published version: http://dx.doi.org/

10.1017/S0143385707000041

Publisher statement: None 


\title{
From local to global analytic conjugacies
}

\author{
XAVIER BUFF $\dagger$ and ADAM L. EPSTEIN $\ddagger$ \\ † Université Paul Sabatier, Laboratoire Emile Picard, 118, Route De Narbonne, \\ 31062 Toulouse Cedex, France \\ (e-mail: buff@picard.ups-tlse.fr) \\ $\$$ Mathematics Institute, University of Warwick, Coventry CV4 7AL, UK \\ (e-mail:adame@maths.warwick.ac.uk)
}

(Received 24 November 2006 and accepted in revised form 9 February 2007)

\begin{abstract}
Let $f_{1}$ and $f_{2}$ be rational maps with Julia sets $J_{1}$ and $J_{2}$, and let $\Psi: J_{1} \rightarrow \mathbb{P}^{1}$ be any continuous map such that $\Psi \circ f_{1}=f_{2} \circ \Psi$ on $J_{1}$. We show that if $\Psi$ is $\mathbb{C}$-differentiable, with non-vanishing derivative, at some repelling periodic point $z_{1} \in J_{1}$, then $\Psi$ admits an analytic extension to $\mathbb{P}^{1} \backslash \mathcal{E}_{1}$, where $\mathcal{E}_{1}$ is the exceptional set of $f_{1}$. Moreover, this extension is a semiconjugacy. This generalizes a result of Julia (Ann. Sci. École Norm. Sup. (3) 40 (1923), 97-150). Furthermore, if $\mathcal{E}_{1}=\emptyset$ then the extended map $\Psi$ is rational, and in this situation $\Psi\left(J_{1}\right)=J_{2}$ and $\Psi^{-1}\left(J_{2}\right)=J_{1}$, provided that $\Psi$ is not constant. On the other hand, if $\mathcal{E}_{1} \neq \varnothing$ then the extended map may be transcendental: for example, when $f_{1}$ is a power map (conjugate to $z \mapsto z^{ \pm d}$ ) or a Chebyshev map (conjugate to $\pm \mathrm{\Psi}_{d}$ with $\mathrm{Ч}_{d}\left(z+z^{-1}\right)=z^{d}+z^{-d}$ ), and when $f_{2}$ is an integral Lattès example (a quotient of the multiplication by an integer on a torus). Eremenko (Algebra i Analiz 1(4) (1989), 102-116) proved that these are the only such examples. We present a new proof.
\end{abstract}

\section{Introduction}

Let $f$ be a rational map of degree two or more. It is well known that if the Julia set $J$ contains a smooth free arc then $J$ is itself a real-analytic arc or Jordan curve. Moreover, in this situation $f$ is semiconjugate to a Blaschke product, and $J$ lies on a round circle or a straight line: in fact,

$$
f \circ \Psi=\Psi \circ B
$$

where $B$ is a Blaschke product and where $\Psi$ is either a Möbius transformation or a quadratic rational map which sends $\mathbb{D}$ and $\mathbb{P}^{1} \backslash \overline{\mathbb{D}}$ conformally onto $\mathbb{P}^{1} \backslash J$. The proof is carried out in two stages: one begins by promoting the given local property to the entire Julia set, and concludes by deducing the global description of the map itself. For details, see [S, Ch. 5].

Our results here are in the same spirit, and our arguments follow a similar outline. The crucial local hypothesis concerns the differentiability, suitably interpreted, of a map 
whose domain may have empty interior. Consider any map $h: Z \rightarrow \mathbb{P}^{1}$ where $Z \subset \mathbb{P}^{1}$, and let $\zeta \in Z$ be any accumulation point. If $\zeta \neq \infty \neq h(\zeta)$ then we say that $h$ is $\mathbb{C}$-differentiable at $\zeta$ if

$$
\lim _{z \rightarrow \zeta} \frac{h(z)-h(\zeta)}{z-\zeta}
$$

exists. Note that the existence of a non-zero finite limit is unaffected by precomposition or postcomposition by local analytic isomorphisms, and in particular by Möbius transformations. Thus, it makes sense to speak of $\mathbb{C}$-differentiability with non-vanishing derivative even when $\zeta$ or $h(\zeta)$ is the point at infinity.

In what follows, we use the term analytic in the sense of Riemann surface theory. As the relevant Riemann surfaces will all be open subsets of $\mathbb{P}^{1}$, our usage of this term is identical with the classical usage of meromorphic.

THEOREM 1. Let $f_{1}$ and $f_{2}$ be rational maps of degrees at least two with Julia sets $J_{1}$ and $J_{2}$, and let $\Psi: J_{1} \rightarrow \mathbb{P}^{1}$ be continuous with $\Psi \circ f_{1}=f_{2} \circ \Psi$ on $J_{1}$. Suppose further that $\Psi$ is $\mathbb{C}$-differentiable, with non-vanishing derivative, at some repelling periodic point $z_{1} \in J_{1}$. Then $\Psi$ admits an analytic extension to $\mathbb{P}^{1} \backslash \mathcal{E}_{1}$, where $\mathcal{E}_{1}$ is the exceptional set of $f_{1}$. Moreover, the extended map $\Psi$ is a semiconjugacy: that is, $\Psi \circ f_{1}=f_{2} \circ \Psi$ on $\mathbb{P}^{1} \backslash \mathcal{E}_{1}$.

Here we recall that the exceptional set of any rational map consists of at most two points, and that any non-empty exceptional set is a superattracting cycle.

The proof of Theorem 1 begins with a preliminary step where we show that the assumption on $\Psi$ implies the existence of an analytic extension to a neighborhood of $z_{1}$. We then promote this local property to successively more global versions: specifically, we first show that $\Psi$ admits an analytic extension to a neighborhood of $J_{1}$, and then argue that $\Psi$ admits a global analytic extension, at least to $\mathbb{P}^{1} \backslash \mathcal{E}_{1}$.

In [J] , Julia studied the equation $\Psi \circ f_{1}=f_{2} \circ \Psi$ under the assumption that $\Psi$ is defined and holomorphic in a neighborhood of a repelling point $z_{1} \in J_{1}$. He first proved that $\Psi$ has a maximal analytic extension defined on a Riemann surface $\Sigma_{1}$ which in general is not a subset of $\mathbb{P}^{1}$. Then, he proved that if $\Sigma_{1}$ is a subset of $\mathbb{P}^{1}$, then $\mathbb{P}^{1} \backslash \mathcal{E}_{1} \subset \Sigma_{1}$ and he gave examples where $\Sigma_{1}=\mathbb{P}^{1} \backslash \mathcal{E}_{1}$ (see the discussion of power maps, Chebyshev maps and Lattès examples below). Finally, he proved that if $f_{1}$ is a Blaschke product and $\Psi$ has an analytic extension to a neighborhood of $J_{1}$, then it has a analytic extension to $\mathbb{P}^{1} \backslash \mathcal{E}_{1}$. Our Theorem 1 asserts that one can get rid of the assumption that $f_{1}$ is a Blaschke product and consider an arbitrary rational map $f_{1}$.

The possibility of an extension to $\mathbb{P}^{1}$ is discussed in Theorem 2 below. This complementary result makes use of the following terminology, which is discussed in more detail in $\S 5$ and [M2].

A power map of degree $d \geq 2$ is a rational map analytically conjugate to $z \mapsto z^{ \pm d}$.

A Chebyshev map of degree $d \geq 2$ is a rational map analytically conjugate to $\pm \mathrm{U}_{d}$ where $\mathrm{\Psi}_{d}$ is the Chebyshev polynomial of degree $d$ defined by

$$
\mathrm{U}_{d}\left(z+\frac{1}{z}\right)=z^{d}+\frac{1}{z^{d}} .
$$


A Lattès example is a rational map $f: \mathbb{P}^{1} \rightarrow \mathbb{P}^{1}$ of degree at least two such that there exists a commutative diagram

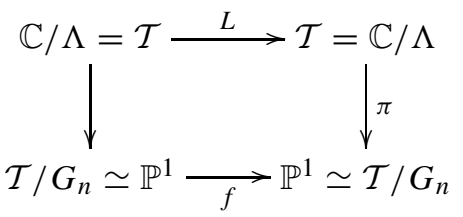

where:

- $\Lambda$ is a discrete additive subgroup of rank two of the complex numbers $\mathbb{C}$;

- $\mathcal{T}=\mathbb{C} / \Lambda$ is the quotient torus;

- $\quad G_{n}$ is the group of $n$th roots of unity acting on $\mathcal{T}$ by rotation around a base point, with $n$ equal to either $2,3,4$ or 6 ;

- $\quad \mathcal{T} / G_{n}$ is the quotient space isomorphic to $\mathbb{P}^{1}$;

- $\quad L: \mathcal{T} \rightarrow \mathcal{T}$ is an affine map which commutes with the action of $G_{n}$.

If the affine map $L$ is of the form $z \mapsto a z+b$ with $a \in \mathbb{Z}$, then we refer to $f$ as an integral Lattès example.

THEOREM 2. Let $f_{1}$ and $f_{2}$ be rational maps of degrees at least two, and let $\Psi: \mathbb{P}^{1} \backslash \mathcal{E}_{1} \rightarrow$ $\mathbb{P}^{1}$ be analytic with $\Psi \circ f_{1}=f_{2} \circ \Psi$ on $\mathbb{P}^{1} \backslash \mathcal{E}_{1}$. Suppose that $\Psi$ does not extend to a rational map $\mathbb{P}^{1} \rightarrow \mathbb{P}^{1}$, so that in particular $\mathcal{E}_{1} \neq \emptyset$. Then $f_{1}$ is either a power map or a Chebyshev map, and $f_{2}$ is an integral Lattès example.

More precisely, there are discrete subgroups $\Gamma \subset \Sigma \subset \operatorname{Isom}(\mathbb{C})$ and an affine map $L: \mathbb{C} \rightarrow \mathbb{C}$ with derivative $a \in \mathbb{Z} \backslash\{-1,0,1\}$ such that the following diagram commutes:

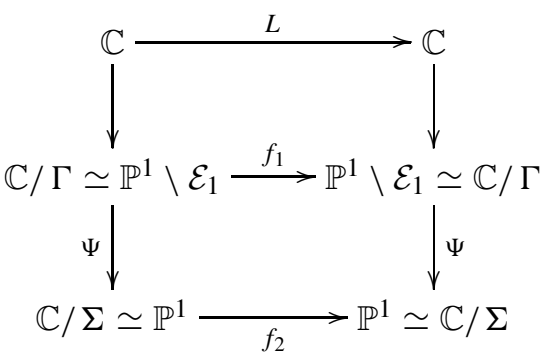

Here $\operatorname{Isom}(\mathbb{C})$ denotes the group of orientation-preserving Euclidean isometries of $\mathbb{C}$, that is, maps of the form $z \mapsto a z+b$ with $|a|=1$. In $\S 5$, we will discuss which discrete subgroups $\Gamma \subset \Sigma \subset \operatorname{Isom}(\mathbb{C})$ and which affine maps $L$ can occur.

Taken together, Theorems 1 and 2, along with well-known properties of rational semiconjugacies, have the following consequence, which is perhaps slightly surprising given the apparently local nature of the assumptions.

COROLlaRY 1. Let $f_{1}$ and $f_{2}$ be rational maps of degrees at least two with Julia sets $J_{1}$ and $J_{2}$, and let $\Psi: J_{1} \rightarrow J_{2}$ be a continuous surjection which semiconjugates the restriction $f_{1}$ to the restriction of $f_{2}$. Assume further that $\Psi$ admits an analytic extension to a neighborhood of some point $z_{1} \in J_{1}$. Then $\Psi$ extends to a rational semiconjugacy from $f_{1}$ to $f_{2}$. Moreover, if $\Psi: J_{1} \rightarrow J_{2}$ is a homeomorphism then $\Psi$ extends to a Möbius conjugacy. 
Remark. Note that it is easy to give examples of transcendental semiconjugacies from degree one rational maps to higher degree rational maps, namely maximally continued inverse linearizing maps associated to repelling cycles and maximally continued inverse repelling Fatou coordinates associated to parabolic cycles.

Remark. After submitting our manuscript, we were informed by the referee that Theorem 2 was shown earlier by Eremenko [E]. We note that our argument differs significantly from Eremenko's. His proof involves the lamellarity (invariance under the flow of a suitable vector field) of the equilibrium measure introduced by Brolin, Lyubich and Freire-LopèsMañe. Our proof uses techniques available at Julia's time. More specifically, we estimate the number of points in lateral orbits for a careful choice of compositions of $f_{2}$ and inverse branches of $f_{2}$. We show that if $f_{1}$ is not a power map or a Chebyshev map or if $f_{2}$ is not a Lattès example, then for a generic point $z \in \mathbb{P}^{1}$, the number of points in $f_{2}^{-n}(z)$ grows faster than $d^{n}$ for any integer $d$. This contradicts the fact that this number is at most $D^{n}$ where $D$ is the degree of the rational map $f_{2}$. The connection with Eremenko's proof presumably lies in the fact that preimages of points equidistribute with respect to the equilibrium measure.

\section{Extension to a neighborhood of the repelling point}

Henceforth, we work under the assumptions of Theorem 1: that is, we assume that $\Psi: J_{1} \rightarrow \mathbb{P}^{1}$ is continuous and $\mathbb{C}$-differentiable, with non-vanishing derivative, at a repelling periodic point $z_{1} \in J_{1}$. Note that if $z_{1}$ is fixed by the iterate $f_{1}^{\circ p}$ then $z_{2}=\Psi\left(z_{1}\right)$ is fixed by the iterate $f_{2}^{\circ p}$. Since the passage to iterates does not affect the Julia set or the exceptional set, we may assume without loss of generality that $f_{i}$ fixes $z_{i}$ for $i=1,2$. Moreover, since the property of $\mathbb{C}$-differentiability with non-vanishing derivative is preserved under locally invertible analytic change of variable, we may further assume that $z_{1}=0=z_{2}$.

After these preliminary reductions, we have $\Psi(z)=c z+o(z)$ as $z \rightarrow 0$, for some $c \in \mathbb{C}^{*}$. Moreover, since

$$
c \cdot f_{1}^{\prime}(0)+o(1)=\frac{\Psi\left(f_{1}(z)\right)}{z}=\frac{f_{2}(\Psi(z))}{z}=f_{2}^{\prime}(0) \cdot c+o(1)
$$

and since $c \neq 0$, the two maps $f_{i}$ have the same multiplier at 0 . Let $\lambda$ be this common multiplier, let $\phi_{i}: U_{i} \rightarrow \mathbb{C}$ be normalized local linearizing maps, so that $\phi_{i} \circ f_{i}(z)=\lambda \phi_{i}(z)$ and $\phi_{i}^{\prime}(0)=1$ for $i=1,2$, and set $\widetilde{\Psi}=\phi_{2} \circ \Psi \circ \phi_{1}^{-1}$. Then $\widetilde{\Psi}(z)=c z+o(z)$ as $z \in \phi_{1}\left(U_{1} \cap J_{1}\right)$ tends to 0 , and moreover $\widetilde{\Psi}(z / \lambda)=\widetilde{\Psi}(z) / \lambda$ for every $z \in \phi_{1}\left(J_{1} \cap U_{1}\right)$. Furthermore, if $z \in \phi_{1}\left(U_{1} \cap J_{1}\right)$ then $z_{n}=z / \lambda^{n}$ also lies in $\phi_{1}\left(U_{1} \cap J_{1}\right)$ for any positive integer $n$, and clearly

$$
\frac{\widetilde{\Psi}\left(z_{n}\right)}{z_{n}}=\frac{\widetilde{\Psi}\left(z / \lambda^{n}\right)}{z / \lambda^{n}}=\frac{\widetilde{\Psi}(z)}{z}
$$

As $n \rightarrow \infty$, we have $z_{n} \rightarrow 0$ and $\widetilde{\Psi}\left(z_{n}\right) / z_{n} \rightarrow c$, and hence $\widetilde{\Psi}(z)=c z$ for every $z \in \phi_{1}\left(U_{1} \cap J_{1}\right)$. In particular, $\widetilde{\Psi}$ extends analytically to a neighborhood of 0 , so the same is true for $\Psi=\phi_{2}^{-1} \circ \widetilde{\Psi} \circ \phi_{1}$. We summarize this discussion as follows. 
LEMMA 1. Let $f_{1}$ and $f_{2}$ be rational maps of degrees at least two with Julia sets $J_{1}$ and $J_{2}$, and let $\Psi: J_{1} \rightarrow \mathbb{P}^{1}$ be continuous with $\Psi \circ f_{1}=f_{2} \circ \Psi$ on $J_{1}$. Suppose further that $\Psi$ is $\mathbb{C}$-differentiable, with non-vanishing derivative, at some repelling periodic point $z_{1} \in J_{1}$. Then $\Psi$ admits an analytic extension to a neighborhood of $z_{1}$.

\section{Extension to a neighborhood of the Julia set}

We now show that $\Psi$ extends analytically to a neighborhood of $J_{1}$.

LEMMA 2. The set of all points $z \in J_{1}$ such that $\Psi$ admits an analytic extension to a neighborhood of $z$ is forward invariant under $f_{1}$.

Proof. Assume that $\Psi$ extends analytically to a neighborhood of $z \in J_{1}$ and set $w=\Psi(z)$. Let $V$ be an open disk containing $w$, and let $U$ be the connected component of $f_{1}^{-1}(V)$ containing $z$. By assumption, if $V$ is sufficiently small then $\Psi$ extends analytically to $U$. Moreover, in this situation the restriction $f_{1}: U \rightarrow V$ is a branched cover, unramified except possibly at $z$. Consequently, there is a cyclic group of analytic isomorphisms $\sigma: U \rightarrow U$, all fixing $z$, such that for $\zeta, \hat{\zeta} \in U$ we have $f_{1}(\zeta)=f_{1}(\hat{\zeta})$ if and only if $\hat{\zeta}=\sigma(\zeta)$ for some such $\sigma$. Furthermore, since $\Psi\left(f_{1}(z)\right)=f_{2}(\Psi(z))$ for all $z \in J_{1}$, it follows that $f_{1}=f_{1} \circ \sigma$ on $U$, and hence

$$
f_{2} \circ \Psi=\Psi \circ f_{1}=\Psi \circ f_{1} \circ \sigma=f_{2} \circ \Psi \circ \sigma
$$

on the infinite set $U \cap J_{1}$. By uniqueness of analytic continuation, it follows that $f_{2} \circ \Psi=f_{2} \circ \Psi \circ \sigma$ on $U$, and hence $f_{2} \circ \Psi: U \rightarrow \mathbb{P}^{1}$ descends to a map which is continuous on $V$ and analytic on $V \backslash\{w\}$, hence analytic on all of $V$, and which extends the given map $\Psi$.

Now let $W$ be a neighborhood of $z_{1} \in J_{1}$ on which $\Psi$ extends analytically. By Montel's theorem, we have $f_{1}^{\circ n}\left(W \cap J_{1}\right)=J_{1}$ for some positive integer $n$. Consequently, it follows from Lemma 2 that $\Psi$ extends analytically to a neighborhood of any given point in $J_{1}$. To obtain the desired extension to a neighborhood of $J_{1}$ we apply the following well-known fact.

LemMa 3. Let $\Psi: J \rightarrow \mathbb{P}^{1}$ be a map from a closed set $J \subseteq \mathbb{P}^{1}$, and suppose that for each $z \in J$ the map $\Psi$ extends analytically to some neighborhood of $z$. Then $\Psi$ extends analytically to a neighborhood of $J$.

Proof. If $J=\mathbb{P}^{1}$ then $\Psi$ is already analytic on all of $\mathbb{P}^{1}$ and there is nothing further to show. Composing with a Möbius transformation if necessary, we may therefore assume that $J$ is a compact set in $\mathbb{C}$.

For $x \in \mathbb{C}$ and $r>0$, we denote by $D_{x}(r)$ the open Euclidean disk with center $x$ and radius $r$. By assumption, for each $x \in J$ there exists $r_{x}>0$ such that $\Psi$ admits an analytic extension $\Psi_{x}$ defined on the disk $U_{x}=D_{x}\left(r_{x}\right)$. Consider the subdisks $V_{x}=D_{x}\left(\frac{1}{3} r_{x}\right)$. Note that if $V_{x} \cap V_{y} \neq \emptyset$ then $|x-y|<\frac{1}{3} r_{x}+\frac{1}{3} r_{y} \leq \frac{2}{3} \max \left(r_{x}, r_{y}\right)$, and hence if $r_{x} \leq r_{y}$ then $V_{x} \subseteq U_{y}$. Moreover, in this situation it follows by uniqueness of analytic continuation that if $J \cap V_{x}$ contains an accumulation point then $\Psi_{x}$ and $\Psi_{y}$ have the same restriction to $V_{x}$, and hence the restrictions $\Psi_{x \mid V_{x}}$ and $\Psi_{y \mid V_{y}}$ agree on $V_{x} \cap V_{y}$. 
As $J \subset \bigcup_{x \in J} V_{x}$, it follows by compactness that $J \subset \bigcup_{j=1}^{N} V_{x_{j}}$ for some $x_{1}, \ldots, x_{N}$ in $J$. We may assume without loss of generality that, for some $n<N$, the intersection $J \cap V_{x_{j}}$ contains an accumulation point precisely for $1 \leq j \leq n$. Since the open set $W=\bigcup_{j=1}^{n} V_{x_{j}}$ contains every accumulation point of $J$, the complement $I=J \backslash W$ consists of finitely many isolated points of $J$. It follows from the observations above that if $1 \leq j, k \leq n$ then $\Psi_{x_{j} \mid V_{x_{j}}}$ and $\Psi_{x_{k} \mid V_{x_{k}}}$ agree on $V_{x_{j}} \cap V_{x_{k}}$; hence the maps $\Psi_{x_{j} \mid V_{x_{j}}}$ for $1 \leq j \leq n$ are all restrictions of some analytic map defined on $W$, and the map so obtained agrees with $\Psi$ on $J \backslash I$. Finally, for the finitely many points $y \in I$, let $W_{y} \ni y$ be pairwise disjoint open disks which are also disjoint from $W$. The map just obtained now extends trivially to an analytic extension of $\Psi$ defined on the open neighborhood $\widehat{W}=W \cup \bigcup_{y \in I} W_{y}$ of $J$.

\section{Extension to the complement of the exceptional set}

Up to now we have shown that $\Psi$ extends analytically to a neighborhood $W$ of $J_{1}$. Here we obtain the desired analytic extension to $\mathbb{P}^{1} \backslash \mathcal{E}_{1}$. Note that since $J_{1}$ is compact, the neighborhood $W$ contains all but finitely many Fatou components. To treat the remaining components we will make use of the following.

LEMmA 4. Let $f_{1}: \Omega \rightarrow \tilde{\Omega}$ be a proper analytic map between open sets $\Omega, \tilde{\Omega} \subseteq \mathbb{P}^{1}$. Let $\Psi: \Omega \rightarrow \mathbb{P}^{1}$ and $f_{2}: \Psi(\Omega) \rightarrow \mathbb{P}^{1}$ be analytic, let $\tilde{\Psi}: U \rightarrow \mathbb{P}^{1}$ be analytic, where $U \neq \emptyset$ is open in $\tilde{\Omega}$, and assume that $\tilde{\Psi} \circ f_{1}=f_{2} \circ \Psi$ on $f_{1}^{-1}(U)$. Then $\tilde{\Psi}$ extends analytically to $\tilde{\Omega}$.

Proof. We claim that, for any point $w \in \tilde{\Omega}$, the value of $f_{2} \circ \Psi(z)$ is independent of the choice of preimage $z \in f_{1}^{-1}(w)$. Note that since $f_{1}$ is an open map, the points $w$ for which this is true form a relatively closed set. Consequently, it suffices to verify the claim on a dense subset of $\tilde{\Omega}$. Since $f_{1}$ has only countably many critical points, there are only countably many critical values, so the points which are not critical values are dense in $\tilde{\Omega}$ : indeed, since $f_{1}: \Omega \rightarrow \tilde{\Omega}$ is proper, the critical values are discrete in $\tilde{\Omega}$. Thus, we may assume without loss of generality that $w$ is not a critical value.

Fix a point $u \in U$ which is not a critical value, and let $\gamma$ be a path in $\tilde{\Omega}$ which connects $u$ to $w$, and which avoids the set of critical values; note that, by the discreteness of critical values, such a path always exists. Now $\tilde{\Psi}$ is defined near $u$, and moreover we have $\tilde{\Psi}=\tilde{\Psi} \circ f_{1} \circ g=f_{2} \circ \Psi \circ g$ for any analytic germ $g$ which is locally inverse to $f_{1}$ near $u$. Since $f_{1}: \Omega \rightarrow \tilde{\Omega}$ is proper, and since there are no critical values on $\gamma$, the germ $g$ admits a unique analytic continuation along that path, leading to an analytic germ $h$ defined near $w$. It follows that the analytic continuation of $\tilde{\Psi}$ along $\gamma$ is the germ $f_{2} \circ \Psi \circ h$. Moreover, the resulting germ is independent of the choice of $\gamma$ : indeed, the analytic continuation of $g$ along any closed path which is based at $u$, and which avoids the critical values, is also locally inverse to $f_{1}$, and hence the result of the corresponding continuation of the germ of $\tilde{\Psi}$ at $u$ is the same germ $\tilde{\Psi}$.

It follows that the prescription $w \mapsto f_{2}(\Psi(z))$, where $z \in \Omega$ is any preimage of $w$, gives a well-defined map $\tilde{\psi}: \tilde{\Omega} \rightarrow \mathbb{P}^{1}$. Moreover, since $f_{1}$ is an open map, it follows that $\tilde{\psi}$ is continuous. Furthermore, by construction $\tilde{\psi}$ is analytic away from the set of critical values, and since the latter constitute a discrete set, it follows by continuity that these are 
removable singularities; hence $\tilde{\psi}$ is analytic on $\tilde{\Omega}$. Since $\tilde{\psi}$ and $\tilde{\Psi}$ agree on $U$, the map $\tilde{\psi}$ is the desired analytic continuation of $\tilde{\Psi}$ to $\tilde{\Omega}$.

Proposition 1. Let $f_{1}$ and $f_{2}$ be rational maps of degrees at least two with Julia sets $J_{1}$ and $J_{2}$. Let $\Psi: J_{1} \rightarrow \mathbb{P}^{1}$ be continuous with $\Psi \circ f_{1}=f_{2} \circ \Psi$ on $J_{1}$, and assume that $\Psi$ admits an analytic extension to a neighborhood of $J_{1}$. Then $\Psi$ admits an analytic extension to $\mathbb{P}^{1} \backslash \mathcal{E}_{1}$, where $\mathcal{E}_{1}$ is the exceptional set of $f_{1}$.

Proof. Assume that $\Psi: W \rightarrow \mathbb{P}^{1}$ is analytic on the open neighborhood $W \supset J_{1}$. Note that, without loss of generality, we may choose $W$ so that every connected component of $W$ intersects $J_{1}$. Consequently, if the restriction $\Psi_{\mid \partial \Omega}$ extends analytically to a Fatou component $\Omega$, then $\Psi$ itself so extends. In view of Lemma 4 and the fact that $W$ must intersect every Fatou component, if $\Psi$ extends analytically to a Fatou component $\Omega$ then $\Psi$ further extends to the image component $f_{1}(\Omega)$. If $\Omega$ is not totally invariant under $f_{1}$ then the backward orbit of $\Omega$ consists of infinitely many Fatou components, and all but finitely many lie in the given neighborhood where $\Psi$ is already analytic. It follows that $\Psi$ extends analytically to every such component $\Omega$.

Suppose now that $\Omega$ is totally invariant under $f_{1}$. Such a component is necessarily a (super)attracting or parabolic basin, with associated fixed point $\zeta$. In this situation we apply a similar argument to an appropriate collection of nested open subsets of $\Omega$. Let $U_{k}=\Omega \backslash f_{1}^{-k}(\bar{D})$, where $D \subset \Omega$ is an open disk chosen as follows. In the (super)attracting case, we take any $D \ni \zeta$ which is small enough that $f_{1}(D)$ lies compactly in $D$; in the parabolic case, we take $D$ to be an attracting petal, so here $\zeta \in \partial D$. By construction, the restriction of $f_{1}$ maps $U_{k+1}$ properly to $U_{k}$, and $U_{k+1} \subset U_{k}$, for every integer $k \geq 0$. Moreover, if $k$ is sufficiently large then $U_{k} \subset W$, and hence $\Psi$ is analytic on such $U_{k}$, and furthermore $\Psi$ semiconjugates $f_{1}$ to $f_{2}$ on $U_{k+1}$. Starting from the restriction of $\Psi$ to such a set $U_{k}$, and applying Lemma 4 inductively, we deduce that the restriction $\Psi_{\mid \partial \Omega}$ admits successive analytic extensions to the sets $U_{j}$ with $j<k$, each such extension giving a semiconjugacy on $U_{j+1}$. In particular, $\Psi_{\mid \partial \Omega}$ extends to an analytic map on $\Omega \backslash \bar{D}$. Furthermore, since $\Omega \backslash \bar{D}$ is connected with the boundary containing $\partial \Omega$, replacing $D$ by a smaller disk yields a further analytic extension. Thus, shrinking $\bar{D}$ to $\zeta$, we obtain an analytic extension of $\Psi_{\mid \partial \Omega}$ to $\Omega \backslash\{\zeta\}$, and hence, as above, it follows that $\Psi$ extends analytically to $\Omega \backslash\{\zeta\}$, giving a semiconjugacy on $\Omega \backslash\{\zeta\}$.

In the parabolic case we have $\zeta \in \partial \Omega$, so there is nothing left to show. If $\zeta \notin \mathcal{E}_{1}$ then there exists a preimage $\xi \neq \zeta$, and $\xi \in \Omega$ by total invariance. If $D$ is sufficiently small then the component $B \ni \xi$ of $f_{1}^{-1}(D)$ is contained in $\Omega \backslash\{\zeta\}$. Since $\Psi \circ f_{1}=f_{2} \circ \Psi$ on $B \backslash\{\xi\}$, it follows from Lemma 4 that $\Psi$ extends analytically to $\Omega$.

\section{Rational semiconjugacies between rational maps}

Here we review certain well-known properties of rational semiconjugacies, and then deduce Corollary 1 from Theorems 1 and 2.

LEMMA 5. Let $f_{1}$ and $f_{2}$ be rational maps with Julia sets $J_{1}$ and $J_{2}$, and let $\Psi$ be a rational map with $\Psi \circ f_{1}=f_{2} \circ \Psi$. If $\Psi$ is not constant then $f_{1}$ and $f_{2}$ have the same degree, and if this degree is two or more then $\Psi\left(J_{1}\right)=J_{2}$ and $\Psi^{-1}\left(J_{2}\right)=J_{1}$. 
Proof. To see that the degrees of $f_{1}$ and $f_{2}$ are equal, observe that

$$
\operatorname{deg} \Psi \cdot \operatorname{deg} f_{1}=\operatorname{deg}\left(\Psi \circ f_{1}\right)=\operatorname{deg}\left(f_{2} \circ \Psi\right)=\operatorname{deg} f_{2} \cdot \operatorname{deg} \Psi
$$

and note that $\operatorname{deg} \Psi \neq 0$ since $\Psi$ is not constant. Assuming now that the common degree is at least two, we first show that $\Psi\left(J_{1}\right) \subseteq J_{2}$, and hence $J_{1} \subseteq \Psi^{-1}\left(J_{2}\right)$, and then show conversely that $\Psi^{-1}\left(J_{2}\right) \subseteq J_{1}$, and hence $J_{2} \subseteq \Psi\left(J_{1}\right)$.

Recall that if $f_{1}$ fixes $z_{1}$ then $f_{2}$ fixes $z_{2}=\Psi\left(z_{1}\right)$. Moreover, it follows from the chain rule that $\lambda_{2}=\lambda_{1}^{\delta}$, where $\lambda_{i}$ are the corresponding multipliers and where $\delta$ is the local degree of $\Psi$ at $z_{1}$; thus, $\Psi$ sends repelling fixed points of $f_{1}$ to repelling fixed points of $f_{2}$. Applying this observation to iterates, we deduce more generally that $\Psi$ sends repelling periodic points of $f_{1}$ to repelling periodic points of $f_{2}$. Since the Julia sets $J_{1}$ and $J_{2}$ are the closures of the corresponding sets of repelling periodic points, it follows that $\Psi\left(J_{1}\right) \subseteq J_{2}$.

Conversely, let $w$ be periodic for $f_{2}$ and let $z$ be any point in the preimage $\Psi^{-1}(z)$. Note that such a point $z$ need not be periodic for $f_{1}$; however, since the forward orbit of $z$ under $f_{1}$ lies in the $\Psi$-preimage of the forward orbit of $w$ by $f_{2}$, such a point $z$ is at least preperiodic. Considering multipliers as above, we deduce that $z$ eventually maps to a repelling periodic cycle. Applying the density of repelling periodic points once again, we conclude that $\Psi^{-1}\left(J_{2}\right) \subseteq J_{1}$.

Note that the same considerations show that a rational semiconjugacy sends attracting periodic points to attracting periodic points of $f_{2}$. In fact, with a bit more care it may be shown that parabolic, indifferently linearizable, and indifferently non-linearizable periodic points are sent to periodic points of the same character.

Proof of Corollary 1. It follows from Theorem 1 that $\Psi$ admits an analytic extension to a semiconjugacy defined on $\mathbb{P}^{1} \backslash \mathcal{E}_{1}$, and we claim that this extension is in fact rational. Indeed, if $\Psi$ has an essential singularity then it follows from Theorem 2 that $\mathcal{E}_{1} \neq \emptyset$, and hence $J_{1}$ is nowhere dense, and moreover that $J_{2}=\mathbb{P}^{1}$. On the other hand, since $\Psi$ is a non-constant analytic map, the image $\Psi\left(J_{1}\right)$ must be a meagre subset of $\mathbb{P}^{1}$, and hence $J_{2}=\Psi\left(J_{1}\right) \neq \mathbb{P}^{1}$ for a contradiction. Thus, $\Psi$ must be a rational semiconjugacy, and hence $\Psi^{-1}\left(J_{2}\right)=J_{1}$ by Lemma 5. Consequently, if $\Psi$ restricts to a homeomorphism between $J_{1}$ and $J_{2}$ then every $z \in J_{2}$ has a unique preimage under $\Psi$. Since $J_{2}$ is infinite, it follows that $\Psi$ has degree one, and hence $\Psi$ is a Möbius transformation.

\section{Some transcendental semiconjugacies between rational maps}

Following Milnor [M2], we will say that a rational map of degree $d \geq 2$ is a power map if it is analytically conjugate to either $p_{d}: z \mapsto z^{d}$ or $p_{-d}: z \mapsto z^{-d}$. The power maps are the rational maps $f$ whose exceptional set $\mathcal{E}_{f}$ contains two points which are either fixed (when it is conjugate to $p_{d}$ ) or exchanged (when it is conjugate to $p_{-d}$ ). The restriction of a power map $f$ to $\mathbb{P}^{1} \backslash \mathcal{E}_{f}$ is a covering map of degree $d$. The Julia set of $p_{ \pm d}$ is the unit circle.

A rational map of degree $d \geq 2$ will be called a Chebyshev map if it is conjugate to $\pm \mathrm{\Psi}_{d}$ where $\mathrm{\Psi}_{d}$ is the degree $d$ Chebyshev polynomial, defined by the equation

$$
\mathrm{\Psi}_{d}\left(z+\frac{1}{z}\right)=z^{d}+\frac{1}{z^{d}} \text {. }
$$


For example,

$$
\mathrm{\Psi}_{2}(z)=z^{2}-2, \quad \mathrm{\Psi}_{3}(z)=z^{3}-3 z \quad \text { and } \quad \mathrm{U}_{4}(z)=z^{4}-4 z^{2}+2 .
$$

The Julia set of $\pm \Psi_{d}$ is the segment $[-2,2]$. The postcritical set of $\pm \Psi_{d}$ consists of the three points $\{-2,2, \infty\}$. If $d$ is even, $-\Psi_{d}$ is conjugate to $\Psi_{d}$ by $-\mathrm{Id}$. However, when $d$ is odd, $\Psi_{d}$ fixes the postcritical set whereas $-\Psi_{d}$ permutes the two points of the postcritical set, and hence cannot be conjugate to $\Psi_{d}$.

The following construction due to Lattès [L] is well-known (see also [M2] for a detailed discussion on Lattès examples). If $\Lambda$ is a discrete group of translations of rank two, then the quotient surface $\mathbb{C} / \Lambda$ is a torus $\mathcal{T}$. Moreover, if $G_{n}$ is the group of $n$th roots of unity acting on $\mathcal{T}$ by rotation around a base point with $n$ equal to either $2,3,4$ or 6 , then the quotient surface $\mathcal{T} / G_{n}$ is a Riemann surface isomorphic to $\mathbb{P}^{1}$. If $L: \mathbb{C} / \Lambda \rightarrow \mathbb{C} / \Lambda$ is an affine map $z \mapsto a z+b$ which commutes with the action of $G_{n}$, then there is an induced rational map $L / G_{n}: \mathcal{T} / G_{n} \rightarrow \mathcal{T} / G_{n}$ from the quotient surface to itself. This rational map has degree $|a|^{2}$, its Julia set is $\mathcal{T} / G_{n}$ and its postcritical set is the set of critical values for the projection $\mathcal{T} \rightarrow \mathcal{T} / G_{n}$

A rational map of degree $D \geq 2$ is said to be a Lattès example if it is conformally conjugate to a map of the form $L / G_{n}: \mathcal{T} / G_{n} \rightarrow \mathcal{T} / G_{n}$. We say that $f$ is an integral Lattès example if $D=d^{2}$ with $d \geq 2$ an integer and $L(z)=a z+b$ with $a= \pm d$. By definition, for such a map $f$ there is a discrete subgroup $\Sigma \subset \operatorname{Isom}(\mathbb{C})$ and an affine map $L: z \mapsto a z+b$ such that the following diagram commutes:

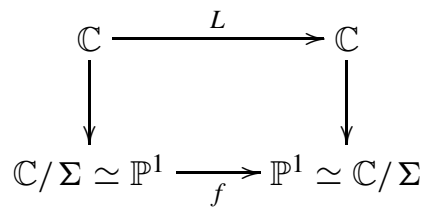

The subgroup $\Lambda \subset \Sigma$ of translations is a normal subgroup of rank two, $\Sigma$ is an extension of $\Lambda$ of degree $n$ and $\Sigma / \Lambda \simeq G_{n}$ acts on the torus $\mathcal{T}=\mathbb{C} / \Lambda$ by rotation around a base point.

Note that if $\Gamma_{1} \subset \Lambda$ is a subgroup of rank one then the projection $\mathbb{C} \rightarrow \mathbb{C} / \Sigma$ factors as $\mathbb{C} \rightarrow \mathbb{C} / \Gamma_{1} \rightarrow \mathbb{C} / \Sigma$. If $f$ is an integral Lattès example, so that $L(z)=a z+b$ with $a \in \mathbb{Z} \backslash\{-1,0,1\}$, then we have the following commutative diagram:

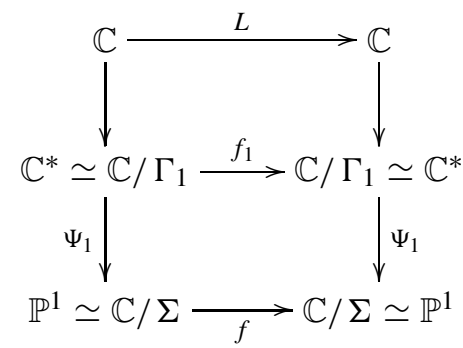

Here $f_{1}$ is a power map analytically conjugate to $z \mapsto z^{a}$. This construction shows the existence of transcendental semiconjugacies between power maps and integral Lattès examples. 
Note that the subgroup $\Lambda$ of $\Sigma$ has index $n$. If $n$ is even, necessarily 2,4 or 6 , then $\Gamma_{1}$ is an index 2 subgroup of some $\Gamma_{2} \subset \Sigma$. The projection $\mathbb{C} \rightarrow \mathbb{C} / \Sigma$ factors as $\mathbb{C} \rightarrow \mathbb{C} / \Gamma_{2} \rightarrow \mathbb{C} / \Sigma$. In this situation, if $f$ is an integral Lattès example, so that $L(z)=a z+b$ with $a \in \mathbb{Z} \backslash\{-1,0,1\}$, then we have the following commutative diagram:

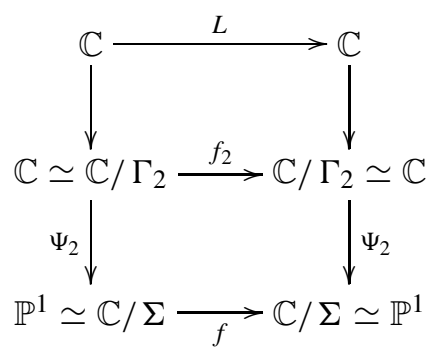

Here $f_{2}$ is a Chebyshev map. If $G_{n}$ acts on $\mathbb{C} / \Lambda$ by rotation around a fixed point of $L$, then $f_{2}$ is conjugate to $\mathrm{\Psi}_{d}$ with $d=|a|$. This is always the case when $a$ is odd. Otherwise, $f_{2}$ is conjugate to $-\Psi_{d}$. This construction shows the existence of transcendental semiconjugacies between Chebyshev maps and integral Lattès examples for which $\Lambda$ has even index in the extension $\Sigma$.

Note that if $\Psi$ is a semiconjugacy between two rational maps $f$ and $g$, then $\Psi \circ f^{\circ k}$ is another such semiconjugacy, for any positive integer $k$. This observation relates to the above discussion as follows.

If $\Gamma_{1} \subset \Gamma_{1}^{\prime}$ are rank-one subgroups of $\Lambda$, then $\Gamma_{1}^{\prime} / \Gamma_{1} \simeq \mathbb{Z} / k \mathbb{Z}$ for some positive integer $k$. The following commutative diagram shows that the semiconjugacy $\Psi_{1}$ associated to $\Gamma_{1}$ may be viewed as $\Psi_{1}^{\prime} \circ f_{1}^{\circ k}$, where $\Psi_{1}^{\prime}$ is the semiconjugacy associated to $\Gamma_{1}^{\prime}$ :

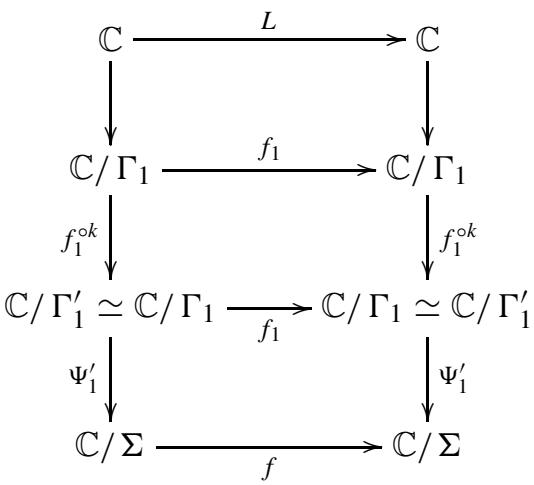

Similarly, if $\Sigma$ contains an involution and if $\Gamma_{2} \subset \Gamma_{2}^{\prime} \subset \Sigma$ are subgroups such that $\Gamma_{1}$ has index 2 in $\Gamma_{2}$ and $\Gamma_{1}^{\prime}$ has index 2 in $\Gamma_{2}^{\prime}$, then the semiconjugacy $\Psi_{2}$ associated to $\Gamma_{2}$ may be viewed as $\Psi_{2}^{\prime} \circ f_{2}^{\circ k}$, where $\Psi_{2}^{\prime}$ is the semiconjugacy associated to $\Gamma_{2}^{\prime}$.

We now present a list of the semiconjugacies which may be obtained through admissible choices of $\Gamma \subset \Sigma \subset \operatorname{Isom}(\mathbb{C})$. In the following section, we will show that up to conjugacies with Möbius transformations, these are the only transcendental semiconjugacies between rational maps of degrees at least two. 
For each Lattès example $f: \mathbb{P}^{1} \rightarrow \mathbb{P}^{1}$ there is an associated 'ramification index' function $r: \mathbb{P}^{1} \rightarrow \mathbb{N}$ which may be characterized as the unique function satisfying the identity

$$
r(f(z))=\operatorname{deg}_{f}(z) \cdot r(z)
$$

for which $r(z)=1$ outside the postcritical set (see, for example, [DH] or [M2]). If $f: \mathbb{P}^{1} \rightarrow \mathbb{P}^{1}$ is conformally conjugate to the quotient map $L / G_{n}: \mathcal{T} / G_{n} \rightarrow \mathcal{T} / G_{n}$ and if $z$ corresponds to $\Theta\left(\tau_{0}\right)$ with $\tau_{0} \in \mathcal{T}$ and $\Theta: \mathcal{T} \rightarrow \mathcal{T} / G_{n} \simeq \mathbb{P}^{1}$ the projection, then $r(z)=\operatorname{deg}_{\Theta}\left(\tau_{0}\right)$. The possible collections of ramification indices are:

- $\quad\{2,2,2,2\}$ if $n=2$;

- $\quad\{3,3,3\}$ if $n=3$;

- $\quad\{2,4,4\}$ if $n=4$; and

- $\quad\{2,3,6\}$ if $n=6$.

We organize the integral Lattès examples into four classes according to which group of $n$th roots of unity acts on the torus $\mathcal{T}=\mathbb{C} / \Lambda$, equivalently on the set of ramification indices. We subdivide each class according to whether the derivative $a$ of the affine map $L$ is even or odd. If $a$ is odd and $n=2$ or $n=4$, we may further distinguish the cases in which $G_{n}$ does, or does not, act on $\mathcal{T}=\mathbb{C} / \Lambda$ by rotation around a fixed point of $L: \mathcal{T} \rightarrow \mathcal{T}$. Finally, in each case, we may describe the postcritical dynamics of $f$ in terms of the ramification indices: for example, $2 \mapsto 6$ means that a point with ramification index 2 is mapped to a point with ramification index 6 . In all cases besides $\{3,3,3\}$, replacing $L$ by $-L$ yields the same Lattès example. We obtain the results in Table 1.

We use the notation $d=|a|$. Recall that when $a$ is even, $\Psi_{d}$ is conjugate to $-\Psi_{d}$ and the dynamics in the postcritical set is

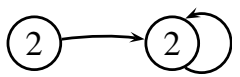

When $a$ is odd we need to distinguish two cases: for $\Psi_{d}$, the dynamics in the postcritical set of $\mathrm{Y}_{d}$ is

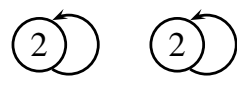

and for $-\Psi_{d}$, the dynamics in the postcritical set of $-\Psi_{d}$ is

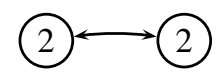

In Table 2, we list the power and Chebyshev maps which are semiconjugate to the various Lattès examples.

\section{Transcendental semiconjugacies}

For the remainder of this paper, we work under the assumptions of Theorem 2: that is, we assume that $\Psi: \mathbb{P}^{1} \backslash \mathcal{E}_{1} \rightarrow \mathbb{P}^{1}$ is a transcendental semiconjugacy from $f_{1}$ to $f_{2}$. In particular, the exceptional set $\mathcal{E}_{1}$ is non-empty, and at least one point in $\mathcal{E}_{1}$ is an essential 
TABLE 1. A classification of Lattès examples.

$\begin{gathered}\text { Ramification } \\ \text { indices }\end{gathered}$
around a fixed point of $L$


TABLE 1. Continued.

\begin{tabular}{|c|c|c|}
\hline $\begin{array}{l}\text { Ramification } \\
\text { indices }\end{array}$ & $\begin{array}{l}G_{n} \text { acts on } \mathcal{T} \text { by rotation } \\
\text { around a fixed point of } L\end{array}$ & $\begin{array}{c}G_{n} \text { does not act on } \mathcal{T} \text { by rotation } \\
\text { around a fixed point of } L\end{array}$ \\
\hline $\begin{array}{c}\{2,3,6\} \\
a=1 \bmod 6 \\
a=5 \bmod 6\end{array}$ & & No Lattès example \\
\hline $\begin{array}{c}\{2,3,6\} \\
a=2 \bmod 6 \\
a=4 \bmod 6\end{array}$ & & No Lattès example \\
\hline $\begin{array}{c}\{2,3,6\} \\
a=3 \bmod 6\end{array}$ & & No Lattès example \\
\hline
\end{tabular}

TABLE 2. Power maps and Chebyshev maps conjugate to Lattès examples.

\begin{tabular}{ccc}
\hline $\begin{array}{c}\text { Ramification } \\
\text { indices }\end{array}$ & $\begin{array}{c}G_{n} \text { acts on } \mathcal{T} \text { by rotation } \\
\text { around a fixed point of } L\end{array}$ & $\begin{array}{c}G_{n} \text { does not act on } \mathcal{T} \text { by rotation } \\
\text { around a fixed point of } L\end{array}$ \\
\hline$\{2,2,2,2\}$ & $p_{d}, p_{-d}, \mathrm{\Psi}_{d}$ & $p_{d}, p_{-d},-\mathrm{\Psi}_{d}$ \\
$\{3,3,3\}$ & $p_{d}$ & $p_{d}$ \\
$a>0$ & & $p_{-d}$ \\
$\{3,3,3\}$ & $p_{-d}$ & $p_{d}, p_{-d},-\mathrm{\Psi}_{d}$ \\
$a<0$ & $p_{d}, p_{-d}, \mathrm{\Psi}_{d}$ & No Lattès example \\
$\{2,4,4\}$ & $p_{d}, p_{-d}, \mathrm{\Psi}_{d}$ & \\
$\{2,3,6\}$ &
\end{tabular}

singularity of $\Psi$. We will first consider the case where $f_{1}$ is a polynomial and $\Psi$ has an essential singularity at infinity. We will then deduce the general case.

Henceforth we write $p$ for $f_{1}$ and $F$ for $f_{2}$. We let $d$ be the degree of $p$ and $D$ the degree of $F$. We denote by $K_{p}$ the filled-in Julia set of $p$, and by $J_{F}$ the Julia set of $F$. Consider the Green's function $G_{p}$ associated to $p$, namely

$$
G_{p}(z)=\lim _{n \rightarrow \infty} \frac{1}{d^{n}} \log ^{+}\left|p^{\circ n}(z)\right| .
$$

Recall that if $\eta$ is greater than

$$
\eta_{p}=\sup _{p^{\prime}(c)=0} G(c)
$$

then the corresponding equipotential

$$
\left\{z \in \mathbb{C}: G_{p}(z)=\eta\right\}
$$


is a Jordan curve: indeed, the normalized Böttcher coordinate bot $_{p}$ extends analytically and univalently to the set $\Omega_{p}$ where $G_{p}$ exceeds $\eta_{p}$, and there we have $G_{p}=\log \left|\operatorname{bot}_{p}\right|$.

Let $\mathbb{H}_{p}$ be the right half-plane

$$
\mathbb{H}_{p}:=\left\{z \in \mathbb{C}: \operatorname{Re}(z)>\eta_{p}\right\}
$$

and define $\Phi: \mathbb{H}_{p} \rightarrow \mathbb{P}^{1}$ by

$$
\Phi:=\Psi \circ \operatorname{bot}_{p}^{-1} \circ \exp .
$$

The map $\operatorname{bot}_{p}^{-1} \circ \exp : \mathbb{H}_{p} \rightarrow \Omega_{p}$ is a universal covering. For all $z \in \mathbb{H}_{p}$,

$$
\Phi(z+2 i \pi)=\Phi(z) \quad \text { and } \quad \Phi(d \cdot z)=F(\Phi(z)) .
$$

In particular, for any integer $k$, we have the following equality:

$$
F^{\circ k}(\Phi(z))=F^{\circ k}\left(\Phi\left(z+2 i \pi d^{-k}\right)\right) .
$$

PROPOSITION 2. Assume that $z_{1} \in \mathbb{H}_{p}$ and $z_{2} \in \mathbb{H}_{p}$ are not critical points of $\Phi$. Then there exists an affine map $A: z \mapsto a z+b$ which sends $z_{1}$ to $z_{2}$ and such that $\Phi \circ A=\Phi$ in a neighborhood of $z_{1}$.

Proof. Since $\Phi$ is locally univalent at $z_{1}$ and $z_{2}$, there exist a neighborhood $U$ of $\Phi\left(z_{1}\right)=$ $\Phi\left(z_{2}\right)$, and neighborhoods $U_{1} \ni z_{1}$ and $U_{2} \ni z_{2}$, such that the restrictions $\Phi: U_{1} \rightarrow U$ and $\Phi: U_{2} \rightarrow U$ are homeomorphisms. We wish to show that the homeomorphism

$$
A:=\left(\left.\Phi\right|_{U_{2}}\right)^{-1} \circ\left(\left.\Phi\right|_{U_{1}}\right): U_{1} \rightarrow U_{2}
$$

is an affine map.

Consider the following vector fields defined on $U$ :

$$
X_{1}:=\left(\left.\Phi\right|_{U_{1}}\right)_{*} \frac{1}{2 i \pi} \frac{d}{d z} \quad \text { and } \quad X_{2}:=\left(\left.\Phi\right|_{U_{2}}\right)_{*} \frac{1}{2 i \pi} \frac{d}{d z} .
$$

If

$$
X_{1}=\xi_{1}(z) \frac{d}{d z} \quad \text { and } \quad X_{2}=\xi_{2}(z) \frac{d}{d z}
$$

then

$$
\left[X_{1}, X_{2}\right]=\left(\xi_{1}(z) \xi_{2}^{\prime}(z)-\xi_{1}^{\prime}(z) \xi_{2}(z)\right) \frac{d}{d z} .
$$

The above commutator vanishes if and only if $X_{1}$ and $X_{2}$ are equal up to multiplication by a constant, in which case the derivative of $A$ is constant. Thus, $A$ is an affine map if and only if $X_{1}$ and $X_{2}$ commute.

Given any open set $V$ compactly contained in $U$, if $\varepsilon$ is sufficiently small, the flows $\phi_{1}(t, z)$ of $X_{1}$ and $\phi_{2}(t, z)$ of $X_{2}$ are defined on $[-\varepsilon, \varepsilon] \times V$ and the maps

$$
\exp \left(t X_{1}\right): z \mapsto \phi_{1}(t, z) \quad \text { and } \quad \exp \left(t X_{2}\right): z \mapsto \phi_{2}(t, z)
$$

are univalent on $V$.

Given univalent maps $f: U \rightarrow \mathbb{C}$ and $g: U \rightarrow \mathbb{C}$, we will denote by $\langle f, g\rangle$ the map $g^{-1} \circ f^{-1} \circ g \circ f$ where defined. 
LEMMA 6. Assume that $f$ and $g$ are analytic in $[-\varepsilon, \varepsilon] \times V$ for some $\varepsilon>0$ and some open set $V \subset \mathbb{C}$, with

$$
f_{s}(z):=f(s, z)=z+s \cdot \xi_{1}(z)+o(s) \quad \text { and } \quad g_{t}(z):=g(t, z)=z+t \cdot \xi_{2}(z)+o(t)
$$

for some holomorphic vector fields $\xi_{1}(z) d / d z$ and $\xi_{2}(z) d / d z$. Then

$$
\left\langle f_{s}, g_{t}\right\rangle(z)=z+s t \cdot \xi_{3}(z)+o(s t)
$$

where $\xi_{3}(z) d / d z=\left[\xi_{1}(z) d / d z, \xi_{2}(z) d / d z\right]$.

Proof. Note that $\left\langle f_{s}, g_{t}\right\rangle$-Id depends analytically on $s$ and $t$ and identically vanishes when $s=0$ or $t=0$. Thus,

$$
\left\langle f_{s}, g_{t}\right\rangle(z)=z+s t \cdot \xi_{3}(z)+o(s t)
$$

for some holomorphic vector field $\xi_{3}$. We have

$$
\begin{aligned}
g_{t} \circ f_{s}(z)= & z+s \cdot \xi_{1}(z)+t \cdot \xi_{2}(z)+s t \cdot \xi_{2}^{\prime}(z) \xi_{1}(z)+o(s)+o(t), \\
f_{s}^{-1} \circ g_{t} \circ f_{s}(z)= & z+s \cdot \xi_{1}(z)+t \cdot \xi_{2}(z)+s t \cdot \xi_{2}^{\prime}(z) \xi_{1}(z) \\
- & s \cdot \xi_{1}(s)-s t \cdot \xi_{1}^{\prime}(z) \xi_{2}(z)+o(s)+o(t) \\
= & z+t \cdot \xi_{2}(z)+s t \cdot \xi_{2}^{\prime}(z) \xi_{1}(z)-s t \cdot \xi_{1}^{\prime}(z) \xi_{2}(z)+o(s)+o(t) \\
\left\langle f_{s}, g_{t}\right\rangle(z)= & z+t \cdot \xi_{2}(z)+s t \cdot \xi_{2}^{\prime}(z) \xi_{1}(z)-s t \cdot \xi_{1}^{\prime}(z) \xi_{2}(z) \\
& -t \xi_{2}(z)+o(s)+o(t) \\
= & z+s t \cdot\left(\xi_{1}(z) \xi_{2}^{\prime}(z)-\xi_{1}^{\prime}(z) \xi_{2}(z)\right)+o(s)+o(t) .
\end{aligned}
$$

It follows that

$$
s t \cdot\left(\xi_{1}(z) \xi_{2}^{\prime}(z)-\xi_{1}^{\prime}(z) \xi_{2}(z)\right)-s t \cdot \xi_{3}=o(s)+o(t)
$$

and hence $\xi_{3}(z) d / d z=\left[\xi_{1}(z) d / d z, \xi_{2}(z) d / d z\right]$.

Given $i_{1}, \ldots, i_{n} \in\{1,2\}$, if $\varepsilon$ is sufficiently small, the map $\psi$ defined by

$$
\psi\left(t_{1}, \ldots, t_{n}, z\right):=\left\langle\left\langle\left\langle\exp \left(t_{1} X_{i_{1}}\right), \exp \left(t_{2} X_{i_{2}}\right)\right\rangle, \ldots\right\rangle, \exp \left(t_{n} X_{i_{n}}\right)\right\rangle(z)
$$

is defined and analytic on $[-\varepsilon, \varepsilon]^{n} \times V$. It follows from the previous lemma and by induction on $n$ that

$$
\psi\left(t_{1}, \ldots, t_{n}, z\right)=z+t_{1} t_{2} \cdots t_{n} \cdot v_{n}(z)+o\left(t_{1} t_{2} \cdots t_{n}\right)
$$

where

$$
v_{n}(z) \frac{d}{d z}=\left[\left[\left[X_{i_{1}}, X_{i_{2}}\right], \ldots\right], X_{i_{n}}\right]=Y_{n} .
$$

We will denote by $\psi_{t}$ the map $z \mapsto \psi(t, \ldots, t, z)$. If $t$ is sufficiently small, then $\psi_{t}$ is analytic and univalent on $V$. Moreover, we have

$$
\psi_{t}(z)=z+t^{n} \cdot v_{n}(z)+o\left(t^{n}\right) .
$$

LEMMA 7. If $Y_{n}$ does not identically vanish on $U$, then there exist $c>0$ and $z_{0} \in U$ such that, for all sufficiently small $t$, the orbit of $z_{0}$ under forward iteration of $\psi_{t}$ contains at least $c / t^{n}$ points. 
Proof. Choose a point $z_{0}$ which is not a zero of $Y_{n}$ and work in a local coordinate around $z_{0}$ in which $Y_{n}=d / d z$. If $r>0$ and $\varepsilon>0$ are sufficiently small, then for all $t \in[-\varepsilon, \varepsilon]$ the map $\psi_{t}$ is defined and univalent on $D\left(z_{0}, r\right)$, with

$$
\left|\psi_{t}(z)-z-t^{n}\right| \leq \frac{1}{2} t^{n} .
$$

Since the image of any point $z \in D\left(z_{0}, r\right)$ lies strictly to the right of $z$, the orbit of $z_{0}$ consists of distinct points. Moreover, it follows by induction that for $j \leq 2 r / 3 t^{n}$ we have

$$
\psi_{t}^{\circ j}\left(z_{0}\right) \in D\left(z_{0}+j t^{n}, j t^{n} / 2\right) \subseteq D\left(z_{0}, r\right)
$$

and hence we may take $c=2 r / 3$.

LEMMA 8. If $k$ is sufficiently large, then $F^{\circ k} \circ \psi_{d^{-k}}=F^{\circ k}$ on $V$.

Proof. We claim that if $W$ is compactly contained in $U$, then for all sufficiently large $k$ we have $F^{\circ k} \circ \phi_{j}\left( \pm d^{-k}, z\right)=F^{\circ k}(z)$ for $j=1,2$ and any $z \in W$. Indeed, if $\Phi\left(z_{1}\right)=z=\Phi\left(z_{2}\right)$ where $z_{1} \in U_{1}$ and $z_{2} \in U_{2}$, then we have

$$
F^{\circ k} \circ \Phi\left(z_{j} \pm 2 i \pi d^{-k}\right)=F^{\circ k} \circ \Phi\left(z_{j}\right)=F^{\circ k}(z)
$$

for $j=1,2$ and every positive integer $k$, so if $k$ is large enough so that $\phi_{j}\left( \pm d^{-k}, z\right)$ is defined, then we have

$$
F^{\circ k} \circ \phi_{j}\left( \pm d^{-k}, z\right)=F^{\circ k} \circ \Phi\left(z_{j} \pm 2 i \pi d^{-k}\right)=F^{\circ k}(z) .
$$

The assertion now follows by a straightforward induction on the size of the relevant commutator.

Consequently, if $Y_{n}$ does not identically vanish on $U$, then for large enough $k$ we may find $c \cdot d^{k n}$ distinct points having the same image by $F^{\circ k}$, and hence $c \cdot d^{k n} \leq D^{k}$, and thus

$$
d^{n} \leq D \quad \text { and } \quad n \leq \frac{\log D}{\log d} .
$$

LEMMA 9. If the vector fields $X_{1}$ and $X_{2}$ do not commute, then for every integer $n \geq 2$ there exist $i_{1}, \ldots, i_{n} \in\{1,2\}$ such that

$$
Y_{n}:=\left[\left[\left[X_{i_{1}}, X_{i_{2}}\right], \ldots\right], X_{i_{n}}\right]
$$

does not identically vanish on $U$.

Proof. We will prove that we can find such a commutator by induction on $n$. The property holds for $n=2$ by the assumption that $X_{1}$ and $X_{2}$ do not commute. Let us assume that $Y_{n}$ has already been produced. Now $Y_{n}$ cannot commute with both $X_{1}$ and $X_{2}$, since otherwise we would have $Y_{n}=c_{i} X_{i}$ for some $c_{i} \neq 0$, in which case $X_{1}$ and $X_{2}$ would commute. If $Y_{n}$ does not commute with $X_{1}$ we choose $Y_{n+1}=\left[Y_{n}, X_{1}\right]$, and otherwise we choose $Y_{n+1}=\left[Y_{n}, X_{2}\right]$.

We thereby deduce that $X_{1}$ and $X_{2}$ commute, and hence $A$ is an affine map. This completes the proof of the proposition. 
Proposition 3. The map $\Phi: \mathbb{H}_{p} \rightarrow \mathbb{P}^{1}$ admits an analytic extension $\widehat{\Phi}: \mathbb{C} \rightarrow \mathbb{P}^{1}$. Moreover, $\widehat{\Phi}\left(z_{1}\right)=\widehat{\Phi}\left(z_{2}\right)$ if and only if there is an isometry $A: \mathbb{C} \rightarrow \mathbb{C}$ sending $z_{1}$ to $z_{2}$ such that $\widehat{\Phi} \circ A=\widehat{\Phi}$.

Proof. Since $\Psi$ has an essential singularity at infinity, there are at most two values which are omitted near infinity. Since $\Phi$ has isolated critical points in $\mathbb{H}_{p}$, the set of critical values is countable. Thus, we may find a point $w \in \mathbb{P}^{1}$ which is not a critical value of $\Phi$, and points $z_{1}, z_{2} \in \mathbb{H}_{p}$ with $\operatorname{Re}\left(z_{2}\right)>\operatorname{Re}\left(z_{1}\right)$ and $\Phi\left(z_{1}\right)=w=\Phi\left(z_{2}\right)$. In view of Proposition 2, there exists an affine map $A: \mathbb{C} \rightarrow \mathbb{C}$ sending $z_{1}$ to $z_{2}$ such that $\Phi \circ A=\Phi$ in a neighborhood of $z_{1}$.

Note that $V=A^{-1}\left(\mathbb{H}_{p}\right) \cap \mathbb{H}_{p}$ is non-empty, since it contains $z_{1}$, and is also connected: indeed, $V$ is either a sector (if $a \notin \mathbb{R}^{-}$) or a strip (if $a \in \mathbb{R}^{-}$). Since $\Phi \circ A=\Phi$ near $z_{1}$, it follows by uniqueness of analytic continuation that $\Phi \circ A=\Phi$ on $V$. There are various cases to consider.

(1) If $A$ is a translation then, since $\operatorname{Re}\left(z_{2}\right)>\operatorname{Re}\left(z_{1}\right)$, the orbit of any point in $\mathbb{C}$ under forward iteration of $A$ eventually lies in $\mathbb{H}_{p}$. Thus, $\Phi$ extends to $\mathbb{C}$ by the formula $\Phi(z)=\Phi \circ A^{\circ n}(z)$, where $n$ is chosen sufficiently large so that $A^{\circ n}(z) \in \mathbb{H}_{p}$.

(2) If $A=a z+b$ with $a \neq 1$ then $A$ has a fixed point $\alpha=b /(1-a)$.

(i) If $\alpha \in \mathbb{H}_{p}$ then $\alpha \in V$, and hence $\Phi \circ A=\Phi$ in a neighborhood of $\alpha$. Exchanging $z_{1}$ and $z_{2}$ and replacing $A$ by $A^{-1}$ if necessary, we may assume without loss of generality that $|a| \leq 1$, so that $V$ contains the forward orbit under $A$ of any point sufficiently close to $\alpha$. Since $\Phi$ is not constant, such an orbit is necessarily finite, and hence $a$ is a root of unity and $A$ is a rational rotation. Consequently, the orbit of any point in $\mathbb{C}$ must intersect $\mathbb{H}_{p}$, and hence $\Phi$ extends to $\mathbb{C}$ by the formula $\Phi(z)=\Phi \circ A^{\circ n}(z)$, where $n$ is chosen so that $A^{\text {on }}(z) \in \mathbb{H}_{p}$.

(ii) If $a<0$ then the fixed point $\alpha$ lies in the segment $\left[z_{1}, z_{2}\right] \subset \mathbb{H}_{p}$. It follows from (i) that $a=-1$, and hence $\Phi$ extends to $\mathbb{C}$.

(iii) If $\alpha \notin \mathbb{H}_{p}$ and $a>0$ then since $\operatorname{Re}\left(z_{2}\right)>\operatorname{Re}\left(z_{1}\right)$ we must have $a>1$, so $A\left(\mathbb{H}_{p}\right) \subseteq \mathbb{H}_{p}$; hence $V=\mathbb{H}_{p}$, and thus $\Phi \circ A=\Phi$ on $\mathbb{H}_{p}$. It follows that $\Phi$ takes the same value at $z$ and the points

$$
A^{-k}\left(A^{k}(z)+2 i \pi\right)=z+\frac{2 i \pi}{a^{k}}
$$

for positive integers $k$. Since these points accumulate in $\mathbb{H}_{p}$, and since $\Phi$ is not constant, this case cannot occur.

(iv) If $\alpha \notin \mathbb{H}_{p}$ and $a \notin \mathbb{R}$ then we may replace $z_{2}$ by $z_{2}+2 i \pi k$ and $A$ by $A_{k}=A+2 i \pi k$, so that the fixed point becomes

$$
\alpha_{k}=\frac{b+2 i \pi k}{1-a} .
$$

If $a \notin \mathbb{R}$ is and $-k \cdot \operatorname{Im}(a)$ is sufficiently large, then $\alpha_{k} \in \mathbb{H}_{p}$. It follows from (i) that $\Phi$ extends to $\mathbb{C}$, and that $A$ is a rational rotation.

We denote by $\widehat{\Phi}$ the analytic extension of $\Phi$ to $\mathbb{C}$. If $\widehat{\Phi}\left(z_{1}\right)=w=\widehat{\Phi}\left(z_{2}\right)$ then, by construction of $\widehat{\Phi}$, for $j=1,2$ there exist isometries $A_{j}$ such that $z_{j}^{\prime}=A_{j}\left(z_{j}\right) \in \mathbb{H}_{p}$ and 
such that $\widehat{\Phi} \circ A_{j}=\widehat{\Phi}$. Now if there is an isometry $A: \mathbb{C} \rightarrow \mathbb{C}$ sending $z_{1}^{\prime}$ to $z_{2}^{\prime}$ such that $\Phi \circ A=\Phi$, then the isometry $A_{2}^{-1} \circ A \circ A_{1}$ sends $z_{1}$ to $z_{2}$, and

$$
\widehat{\Phi} \circ A_{2}^{-1} \circ A \circ A_{1}=\Phi \circ A \circ A_{1}=\Phi \circ A_{1}=\widehat{\Phi}
$$

near $z_{1}$, and hence everywhere. Thus, we may assume without loss of generality that $z_{1}$ and $z_{2}$ belong to $\mathbb{H}_{p}$.

In any neighborhood of $w$, we may find a point $w^{*}$ which is not a critical value of $\Phi$ and which has a preimage $z_{1}^{*} \in \mathbb{H}_{p}$ near $z_{1}$ and a preimage $z_{2}^{*} \in \mathbb{H}_{p}$ near $z_{2}$. Moreover, if $w^{*}$ is sufficiently close to $w$ then we may arrange that $z_{1}$ is the preimage of $w$ closest to $z_{1}^{*}$, and that $z_{2}$ is the preimage of $w$ closest to $z_{2}^{*}$. Since neither $z_{1}^{*}$ nor $z_{2}^{*}$ are critical points of $\Phi$, there exists an isometry $A$ sending $z_{1}^{*}$ to $z_{2}^{*}$ such that $\Phi \circ A=\Phi$. This isometry permutes the preimages of $w$, and sends the one closest to $z_{1}^{*}$ to the one closest to $z_{2}^{*}$. Thus, we have $A\left(z_{1}\right)=z_{2}$, and hence $A$ is the required isometry.

Let $\Sigma$ be the group of isometries $A: \mathbb{C} \rightarrow \mathbb{C}$ such that $\Phi \circ A=\Phi$. Each such isometry acts as a permutation of $\Phi^{-1}(w)$, for any $w \in \mathbb{P}^{1}$; note that if such a set is non-empty it is necessarily infinite, as $\Phi$ commutes with translation by $2 i \pi$. Since the preimage of any point $w \in \mathbb{P}^{1}$ is a discrete set, and since any point $z \in \mathbb{C}$ is fixed by only finitely many elements of $\Sigma$, it follows that $\Sigma$ is a discrete group. We denote by $\pi: \mathbb{C} \rightarrow \mathbb{C} / \Sigma$ the canonical projection from $\mathbb{C}$ to the orbifold $\mathbb{C} / \Sigma$.

Since for any points $z_{1}$ and $z_{2}$ such that $\Phi\left(z_{1}\right)=\Phi\left(z_{2}\right)$ there exists $A \in \Sigma$ sending $z_{1}$ to $z_{2}$, it follows that $\widehat{\Phi}: \mathbb{C} \rightarrow \mathbb{P}^{1}$ induces an injective map $\iota: \mathbb{C} / \Sigma \rightarrow \mathbb{P}^{1}$ such that the following diagram commutes:

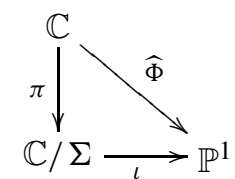

Moreover, since $\Phi(d \cdot z)=F(\Phi(z))$ on $\mathbb{H}_{p}$, we also have the following commutative diagram:

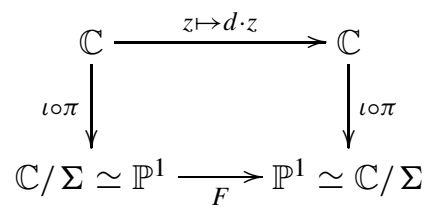

Proposition 4. The rational map $F$ is a Lattès example of degree $D=d^{2}$ induced by multiplication by $d$ on a torus.

Proof. We have seen that $\Sigma$ is a discrete group of isometries of $\mathbb{C}$. Let $\Lambda \subseteq \Sigma$ be the subgroup consisting of translations. Note that $\Lambda$ contains the translation by $2 i \pi$. We claim that $\Lambda$ has rank two. Indeed, if $\Sigma$ were of rank one then:

- $\quad$ either $\sigma$ would be generated by $z \mapsto z+2 i \pi$, and each point $w \in \mathbb{P}^{1}$ would have at most one preimage by $\Phi$ modulo $2 i \pi$;

- $\quad$ or $\sigma$ would be generated by $z \mapsto z+2 i \pi$ and some order two rotation, and each point $w \in \mathbb{P}^{1}$ would have at most two preimages by $\Phi$ modulo $2 i \pi$. 
However, using Picard's theorem we have seen that there are points $w \in \mathbb{P}^{1}$ whose preimages under $\Phi$ have arbitrarily large real parts.

Since $\Sigma$ has rank two, it follows that $\mathbb{C} / \Lambda$ is a torus $\mathcal{T}$. One may show (see, for example, [M2]) that $G=\Sigma / \Lambda$ is a cyclic group of order $n \in\{2,3,4,6\}$, which acts on $\mathcal{T}$ by rotation around a base point. Multiplication by $d$ in $\mathbb{C}$ induces an affine map $L: \mathcal{T} \rightarrow \mathcal{T}$ which commutes with the action of $G$. Moreover, there is an analytic map $\Theta: \mathcal{T} \rightarrow \mathbb{P}^{1}$, necessarily of finite degree, such that the following diagram commutes:

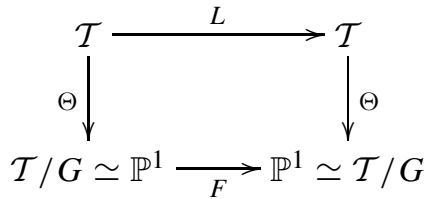

It follows that $F$ is a Lattès example. Since $F$ is induced by multiplication by $d$ on the torus $\mathcal{T}$, the degree of $F$ is $D=d^{2}$.

PROPOSITION 5. The polynomial $p$ is conjugate to either $z \mapsto z^{d}$ or to $\pm \Psi_{d}$. Moreover, if $p$ is conjugate to $\pm \mathrm{\Psi}_{d}$ then $n \neq 3$.

Proof. We may assume without loss of generality that $p$ is not conjugate to a power map, and hence $\Psi$ is defined on $\mathbb{C}$, and every point $z \in \mathbb{C}$ has infinite backward orbit under $p$.

Consider the ramification function $r_{F}: \mathbb{P}^{1} \rightarrow \mathbb{N}$ associated to the Lattès map $F$. Recall that $r_{F}$ takes the value 1 outside of the postcritical set, and that

$$
r_{F}(F(w))=\operatorname{deg}_{F}(w) \cdot r_{F}(w)
$$

for every $w \in \mathbb{P}^{1}$. These properties determine $r_{F}$ uniquely (for details, see $[\mathbf{D H}],[\mathbf{M}]$ or [M2]). Note further that

$$
r_{F}(\widehat{\Phi}(z))=\operatorname{deg}_{\widehat{\Phi}}(w)
$$

for every $z \in \mathbb{C}$. Now consider the function $r_{p}: \mathbb{C} \rightarrow \mathbb{Q}$ given by

$$
r_{p}(z)=\frac{r_{F}(\Psi(z))}{\operatorname{deg}_{\Psi}(z)} .
$$

Since $F \circ \Psi=\Psi \circ p$, we have

$\operatorname{deg}_{F}(\Psi(z)) \cdot \operatorname{deg}_{\Psi}(z) \cdot r_{p}(z)=r_{F}(F \circ \Psi(z))=r_{F}(\Psi \circ p(z))=\operatorname{deg}_{\Psi}(p(z)) \cdot r_{p}(p(z))$

and

$$
\operatorname{deg}_{F}(\Psi(z)) \cdot \operatorname{deg}_{\Psi}(z)=\operatorname{deg}_{\Psi}(p(z)) \cdot \operatorname{deg}_{p}(z)
$$

and hence

$$
r_{p}(p(z))=\operatorname{deg}_{p}(z) \cdot r_{p}(z) .
$$

Note that if $r_{p}(z) \neq 1$ then $r_{F}(\Psi(z)) \neq 1$ or $\operatorname{deg}_{\Psi}(z) \neq 1$. Thus, the points where $r_{p}$ differs from 1 are either critical points of $\Psi$ or preimages of the finitely many points where $r_{F}$ differs from 1 ; note that any bounded region contains only finitely many such points. Since any point $z \in \mathbb{C}$ has infinite backward orbit under $p$, there exist backward orbit points where $r_{p}$ takes the value 1 . It follows that $r_{p}$ is an integer-valued function. 
The map $\operatorname{bot}_{p}^{-1} \circ \exp$ is locally univalent near any $z \in \mathbb{H}_{p}$, so if $w=\operatorname{bot}_{p}^{-1} \circ \exp (z)$ then

$$
r_{F}(\Psi(z))=r_{F}(\Phi(z))=\operatorname{deg}_{\Phi}(z)=\operatorname{deg}_{\Psi}(w) .
$$

It follows that $r_{p}$ is identically 1 in the region where $G_{p}$ exceeds $\eta_{p}$. Consequently, the set $S=\left\{z \in \mathbb{C}: r_{p}(z)>1\right\}$ is finite. Note that $S$ contains the postcritical set $\mathcal{P}_{p} \subset \mathbb{C}$ of $p$, by equation (1) and the fact that $r_{p}$ is integer valued. Thus, $p$ is postcritically finite. Moreover, as the only way to have $r_{p}(z)=1$ and $r_{p}(p(z))>1$ is for $z$ to be a critical point of $p$, we in fact have $S=\mathcal{P}_{p}$, and furthermore

$$
p^{-1}\left(\mathcal{P}_{p}\right)=\mathcal{P}_{p} \cup \mathcal{C}_{p}
$$

where $\mathcal{C}_{p} \subset \mathbb{C}$ denotes the set of (finite) critical points of $p$. Applying the RiemannHurwitz formula to $p: \mathbb{C} \backslash\left(\mathcal{P}_{p} \cup \mathcal{C}_{p}\right) \rightarrow \mathbb{C} \backslash \mathcal{P}_{p}$, we deduce that

$$
1-\operatorname{card}\left(\mathcal{P}_{p} \cup \mathcal{C}_{p}\right)=d \cdot\left(1-\operatorname{card}\left(\mathcal{P}_{p}\right)\right) .
$$

Since $p$ has $d-1$ critical points counting multiplicity, we have $k \leq d-1$ where $k$ is the number not counting multiplicity. Setting $\ell=\operatorname{card}\left(\mathcal{P}_{p}\right) \geq 1$, we deduce from equation (2) that $1-(\ell+m) \leq d \cdot(1-\ell)$, and hence $(d-1)(\ell-1) \leq m \leq d-1$. Thus, we must have $\ell \leq 2$.

If $\ell=1$, equation (2) implies that $\operatorname{card}\left(\mathcal{P}_{p} \cup \mathcal{C}_{p}\right)=1$, and hence $p$ has only one critical point, and this point is fixed by $p$. But then $p$ is conjugate to $z \mapsto z^{d}$, contrary to assumption. Thus, we must have $\ell=2$, and hence equation (2) implies that $\operatorname{card}\left(\mathcal{P}_{p} \cup \mathcal{C}_{p}\right)=d+1$. It follows that $p$ has $d-1$ simple critical points, none of which is postcritical. Conjugating with an affine map if necessary, we may assume that $\mathcal{P}_{p}=\{-2,2\}$.

Let $\pi: \mathbb{P}^{1} \rightarrow \mathbb{P}^{1}$ be the degree-two ramified covering given by

$$
\pi(z)=z+\frac{1}{z} .
$$

Note that $\pi$ has critical points at -1 and 1 , with images -2 and 2 , and that $\pi$ : $\mathbb{P}^{1} \backslash\{-1,1\} \rightarrow \mathbb{P}^{1} \backslash\{-2,2\}$ is a covering map of degree two. The map $p \circ \pi: \mathbb{P}^{1} \rightarrow \mathbb{P}^{1}$ has simple critical points at all the preimages of +1 and -1 , along with $d-1$ critical points at 0 and $d-1$ critical points at $\infty$. It follows that there exists a (unique) lift $\tilde{p}$ fixing $\infty$ such that the following diagram commutes:

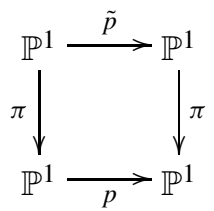

The degree $d$ rational map $\tilde{p}: \mathbb{P}^{1} \rightarrow \mathbb{P}^{1}$ has $d-1$ critical points at the fixed point $\infty$, and hence $\tilde{p}$ polynomial. Since the preimages of 0 by $\tilde{p}$ are preimages of $\infty$ by $p \circ \pi$, we deduce that $\tilde{p}^{-1}(0)=\{0\}$, and hence we must have $\tilde{p}(z)=a z^{d}$ for some $a \in \mathbb{C}^{*}$. Finally, since $\pi(\tilde{p}(1))=p \circ \pi(1) \in \mathcal{P}_{p}=\{-2,2\}$, it follows that $\tilde{p}(1)= \pm 1$, and hence $\tilde{p}(z)= \pm z^{d}$. If $\tilde{p}(z)=z^{d}$, then $p=\mathrm{Ч}_{d}$, and otherwise $p=-\mathrm{Ч}_{d}$. 
To complete the proof of the proposition, note than if $p$ is conjugate to $\pm \mathrm{U}_{d}$ then $r_{p}( \pm 2)=2$, and hence $r_{F}(\Psi( \pm 2))$ must be even. On the other hand, if $n=3$ then $r_{F}$ takes the value 1 at all but three points where the value is 3 (see [M2]).

As a corollary of Propositions 4 and 5, we deduce that if $f_{1}$ and $f_{2}$ are rational maps related by a transcendental semiconjugacy $\Psi: \mathbb{P}^{1} \backslash \mathcal{E}_{1} \rightarrow \mathbb{P}^{1}$, and if $f_{1}$ fixes $\mathcal{E}_{1}$ pointwise, then $f_{1}$ is a power map or a Chebyshev map and $f_{2}$ is a Lattès example. To complete the proof of Theorem 2, we must consider the case where $f_{1}$ exchanges the exceptional points: note that up to Möbius conjugacy we then have $f_{1}(z)=z^{-d}$ for some integer $d \geq 2$.

PROPOSITION 6. Let $F$ be a rational map. If there exists a transcendental semiconjugacy $\Psi: \mathbb{C}^{*} \rightarrow \mathbb{P}^{1}$ between the power map $z \mapsto z^{-d}$ and $F$, then $F$ is a Lattès example induced by an affine map with derivative $-d$.

Proof. Let $p$ be the power map $z \mapsto z^{-d}$. Since $\Psi$ is a transcendental semiconjugacy between $p^{\circ 2}$ and $F^{\circ 2}$, we have the following commutative diagrams:

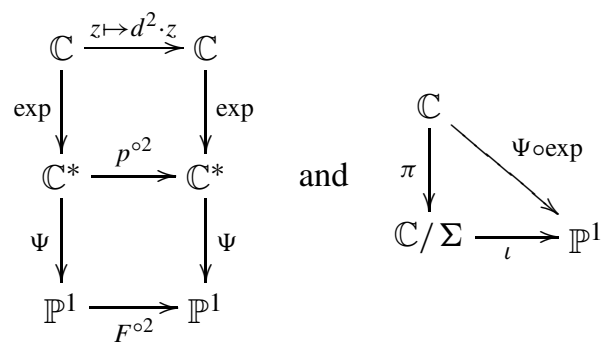

Here $\Sigma$ is a discrete group of isometries of $\mathbb{C}$, the map $\pi: \mathbb{C} \rightarrow \mathbb{C} / \Sigma$ is the canonical projection, and the map $\iota: \mathbb{C} / \Sigma \rightarrow \mathbb{P}^{1}$ is an isomorphism. Since exp : $\mathbb{C} \rightarrow \mathbb{C}$ conjugates multiplication by $-d$ to $p$ and since $\Psi$ semiconjugates $p$ to $F$, we also have the following commutative diagram:

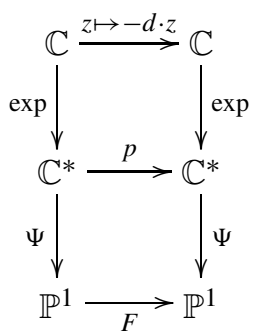

It follows that $F$ is a Lattès example induced by an affine map with derivative $-d$.

Proposition 7. Let $F$ be a Lattès example of type $\{3,3,3\}$.

- If there exists a transcendental semiconjugacy between $z \mapsto z^{d}$ and $F$, then $F$ is not induced by an affine map with negative derivative.

- If there exists a transcendental semiconjugacy between $z \mapsto z^{-d}$ and $F$, then $F$ is not induced by an affine map with positive derivative.

Proof. Let $a$ be the derivative of an affine map inducing $F$. We claim that the fixed point multipliers of $F$ belong to the set $\left\{a, e^{2 i \pi / 3} a, e^{-2 i \pi / 3} a, a^{3}\right\}$. Indeed, if $z \in \mathbb{P}^{1}$ is a fixed 
point of $F$ and $\tau \in \mathbb{C}$ lies above $z$, then $L(\tau)$ also lies above $z$. Thus, we must have $\sigma \circ L(\tau)=\tau$ for some isometry $\sigma \in \Sigma$. The derivative of $\sigma \circ L$ at $\tau$ necessarily belongs to the set $\left\{a, e^{2 i \pi / 3} a, e^{-2 i \pi / 3} a\right\}$. Now the projection $\mathbb{C} \rightarrow \mathbb{C} / \Sigma$ semiconjugates $\sigma \circ L$ to $F$. If $\tau$ is not a critical point of the projection then the multiplier of $F$ at $z$ is equal to the multiplier of $\sigma \circ L$ at $\tau$. On the other hand, if $\tau$ is a critical point of the projection, then the projection has local degree three at $\tau$, and hence the multiplier of $F$ at $z$ is $a^{3}$.

Now if $z \mapsto z^{\delta}$ is semiconjugate to $F$, where $\delta= \pm d$, then $|a|=d=|\delta|$. Moreover, any fixed point $z \in \mathbb{C}^{*}$ of the power map has multiplier $\delta$. The image under the semiconjugacy is a fixed point of $F$ with multiplier $\delta^{m}$, where $m$ is the local degree of the semiconjugacy at $z$. Consequently, we must have $a=\delta$.

Acknowledgement. We thank Lasse Rempe for helping to simplify our original argument.

\section{REFERENCES}

[DH] A. Douady and J. H. Hubbard. A proof of Thurston's topological characterization of rational functions. Acta Math. 171(2) (1993), 263-297.

[E] A. Eremenko. Some functional equations connected with the iteration of rational functions. Algebra $i$ Analiz 1(4) (1989), 102-116 (transl. Leningrad Math. J. 1(4) (1990), 905-919).

[J] G. Julia. Sur une classe d'équations fonctionnelles. Ann. Sci. École Norm. Sup. (3) 40 (1923), 97-150.

[L] L. Lattès. Sue l'itération des substitutions rationnelles et les fonctions de Poincaré. C. R. Acad. Sci. Paris 166 (1918) 26-28.

[M] J. Milnor. Dynamics in One Complex Variable, Introductory Lectures. Friedr. Vieweg \& Sohn, Braunschweig, 1999.

[M2] J. Milnor. On Lattès maps. Dynamics on the Riemann Sphere. Eds. P. Hjorth and C. L Petersen. A Bodil Branner Festschrift, European Mathematical Society, 2006.

[S] N. Steinmetz. Rational Iteration; Complex Analytic Dynamical Systems (de Gruyter Studies in Mathematics, 16). Walter de Gruyter, Berlin, 1993. 